\title{
A Pattern-based Imaging Approach to Pediatric Jaw Lesions
}

\author{
Biswanath Sahu ${ }^{1}$ Rama Anand ${ }^{1}$ Sandeep Kumar ${ }^{1}$ \\ Manjula Jain ${ }^{3}$ \\ ${ }^{1}$ Department of Radio-Diagnosis, Lady Hardinge Medical College \\ and Associated Hospitals, New Delhi, India \\ ${ }^{2}$ Department of Dental and Oral Surgery, Lady Hardinge Medical \\ College and Associated Hospitals, New Delhi, India \\ ${ }^{3}$ Department of Pathology, Lady Hardinge Medical College and \\ Associated Hospitals, New Delhi, India

Ravi Shankar Solanki ${ }^{1}$ Pravesh Mehra²

\begin{abstract}
Address for correspondence Prof. Rama Anand, MD, Department of Radio-Diagnosis, Lady Hardinge Medical College and Associated Hospitals, Shaheed Bhagat Singh Marg, New Delhi 110001, India (e-mail: rama_home@yahoo.com).
\end{abstract}

\begin{abstract}
Keywords

- computed tomography

- jaw lesion

- nonodontogenic

- odontogenic

- orthopantomograph

- pediatric

Jaw lesions in the pediatric population, although infrequently encountered in clinical practice, can cause functional impairment and cosmetic disfiguring. It is further complicated by the difficulty in diagnosis due to complex anatomy and facial developmental process during infancy and childhood. Intraosseous pediatric jaw lesions may vary from odontogenic to nonodontogenic types with nonspecific clinical features in most cases. They deserve careful attention by a systematic approach to provide a relevant diagnosis or differential diagnosis for timely management. Imaging plays a major role in diagnosis with orthopantomograph being the foremost investigation, followed by cross-sectional imaging, essentially computed tomography as a problem-solving tool. This article highlights the imaging spectrum of various jaw lesions in the pediatric population with a pattern-based approach for radiological diagnosis.
\end{abstract}

\section{Introduction}

Various physiological changes with rapid growth and development process in the maxillofacial area during the mixed dentition period in children affect the growth potential of tumors and tumorlike lesions with considerable significant morbidity. ${ }^{1}$ As in adults, pediatric jaw lesions can arise from odontogenic or nonodontogenic tissues; however, the majority of pediatric jaw lesions are nonodontogenic and benign in nature. The high prevalence of dental disease in children results in inflammatory, infective, and reactive processes having substantial overlap in imaging features with more serious locally aggressive tumors or malignant conditions, thereby requiring invasive tissue diagnosis for proper management in some cases. ${ }^{1,2}$

The radiological diagnosis of various jaw lesions is based on the patient's history, location of the lesion, and characteristic radiographic alteration of the lesion as well as adjacent structures. Multiple imaging modalities, like orthopantomograph (OPG), multidetector computed tomography (MDCT), cone beam $\mathrm{CT}(\mathrm{CBCT})$, and magnetic resonance imaging (MRI) are available for the workup of jaw lesions., ${ }^{2,3}$

MRI is a useful adjunct to CT, because of its superior soft-tissue contrast, better delineation of internal components of a lesion, and ability to assess the involvement of the bone marrow in infectious and malignant processes affecting the jaw. ${ }^{1,3}$

Since information obtained from CT and MRI is often complementary, the use of a modality is also governed by institutional practice and availability. Moreover, some of the disadvantages like long scan time, need for sedation of small children, and high cost limit the use of MRI. published online

May 31, 2021
DOI https://doi.org/

$10.1055 / \mathrm{s}-0041-1729767$

ISSN 0971-3026 (c) 2021. Indian Radiological Association

This is an open access article published by Thieme under the terms of the Creative Commons Attribution-NonDerivative-NonCommercial-License, permitting copying and reproduction so long as the original work is given appropriate credit. Contents may not be used for commercial purposes, or adapted, remixed, transformed or built upon. (https://creativecommons.org/licenses/by-nc-nd/4.0/).

Thieme Medical and Scientific Publishers Private Ltd. A-12, Second Floor, Sector -2, NOIDA -201301, India 
Although imaging findings may not lead to a specific diagnosis, they do help narrow the differential diagnosis and guide further workup. Certain lesions like aneurysmal bone cyst $(A B C)$ with characteristic diagnostic imaging appearance do not need biopsy, whereas for other lesions, imaging may help select the appropriate biopsy site., ${ }^{1,2}$

This article provides a systematic comprehensive imaging approach essentially using the OPG and MDCT features along with clinical presentation to provide the diagnosis or appropriate differential diagnosis of jaw lesions in patients younger than 18 years.

The lesions of the jaw are classified broadly into cysts and tumors of the jaw with further subclassifications into their odontogenic and nonodontogenic counterparts. The latest edition of the classification of odontogenic and maxillofacial bone tumors was published by World Health Organization (WHO) in 2017.4.5 - Table 1 shows the classification of odontogenic cysts and tumors of the jaw modified from the updated WHO classification.

\section{Approach to Imaging Evaluation of Jaw Lesions in Children}

The pediatric jaw contains 20 teeth, 10 each in the maxilla and mandible, with lack of premolars and third molars. Two important components of tooth are the crown (enamel-covered superior portion of the tooth) and the root (inferior portion covered with the cementum). Tooth-related jaw lesions are termed "pericoronal," if associated with the crown of the tooth and "periapical" if it is around the apex of the root. ${ }^{6}$

- The first step in the evaluation of jaw lesions is taking the clinical history and identifying the age of the patient.

- The next step is to look for the anatomical location of the lesion within the maxilla or the mandible and relationship of the lesion to the adjacent tooth and the inferior alveolar canal. The predilection of a lesion to occur at a particular site in the jaw also helps narrow the differential diagnosis. ${ }^{7-9}$ The lesions seen above the inferior alveolar canal are usually odontogenic, whereas nonodontogenic lesions are seen below it. Rarely, widening of the inferior alveolar canal may represent lesions of vascular or neural origin. ${ }^{7}$

- Radiologically, jaw lesions are grouped into three categories on the basis of density: radiolucent, radiopaque, and mixed-density lesions. More than $80 \%$ of lesions are radiolucent, whereas most mixed-density lesions are attributed to fibro-osseous lesions, inflammatory or malignant etiologies. ${ }^{7}$

- For an odontogenic lesion, the exact location of the lesion with reference to the tooth, whether pericoronal or periapical, is to be looked for.

- Characteristics on imaging, such as lesion border, locularity, zone of transition, and multiplicity, are of utmost importance for diagnostic differentiation. A well-marginated unilocular radiolucent lesion is likely due to a slow-growing benign process. However, a multilocular lesion with defined margins and narrow transition zone may represent an aggressive benign process. ${ }^{7,8}$
Table 1 Classification of odontogenic cysts and tumors of jaw (Modified from WHO 2017 classification) 4,5 $^{4}$

\begin{tabular}{|l|}
\hline Odontogenic cysts \\
\hline Odontogenic inflammatory cysts \\
\hline Radicular cyst \\
\hline Collateral inflammatory cysts \\
\hline Odontogenic developmental cyst \\
\hline Dentigerous cyst \\
\hline Odontogenic keratocyst \\
\hline Lateral periodontal and botryoid odontogenic cyst \\
\hline Gingival cyst \\
\hline Glandular odontogenic cyst \\
\hline Calcifying odontogenic cyst \\
\hline Orthokeratinized odontogenic cyst \\
\hline Odontogenic tumors \\
\hline Benign epithelial odontogenic tumors: \\
\hline Ameloblastoma (conventional, unicystic, extraosseous/ \\
\hline peripheral, and metastasizing/malignant type) \\
\hline Squamous odontogenic tumor \\
\hline Adenomatoid odontogenic tumor \\
\hline Calcified epithelial odontogenic tumor \\
\hline Benign mesenchymal odontogenic tumors: \\
\hline Odontogenic myxoma or myxofibroma \\
\hline Odontogenic fibroma \\
\hline Cementoblastoma \\
\hline Cemento-ossifying fibroma \\
\hline Benign mixed epithelial and mesenchymal odontogenic tumors \\
\hline Odontoma (compound and complex type) \\
\hline Ameloblastic fibroma \\
\hline Primordial odontogenic tumor \\
\hline Dentinogenic ghost cell tumor \\
\hline Malignant odontogenic tumors (not seen in children) \\
\hline
\end{tabular}

- Other important imaging features that help in characterization of jaw lesions are bone expansion, cortical integrity, periosteal reaction, perilesional halo, growth pattern of the lesion whether along the long axis or perpendicular to jaw bones, and effect on surrounding structures and associated soft tissue. Displacement of teeth generally represents a slow-growing lesion. In addition, slow-growing benign processes usually result in directional resorption of tooth secondary to pressure effects, whereas malignant lesions can often show a nondirectional resorption of teeth due to destruction of the lamina dura. ${ }^{7}$

Typical characteristics of benign jaw lesion on CT are well-defined borders with a narrow zone of transition, cortical expansion and/or cortical bowing, displacement of adjacent structures, and directional root resorption. In malignant lesions, characteristic CT features are ill-defined margins, wide transition zone, cortical breach, periosteal reaction, and associated soft tissue. However, certain aggressive infective 
and inflammatory lesions may also show associated soft tissue due to infiltration of adjacent structures with nondirectional root resorption. ${ }^{6,7}$ - Fig. 1 shows the imaging approach to evaluation of jaw lesions in children.

Imaging features of some common, uncommon neoplastic and nonneoplastic jaw lesions in the pediatric population are summarized in this article with emphasis on key diagnostic features.

\section{Odontogenic Lesions}

Odontogenic lesions typically involve only one tooth or a specific part of the tooth and are usually above the inferior alveolar canal. They do not generally cause pain due to their slow-growth tendency and therefore are often detected incidentally.

\section{Radiolucent Lesions}

\section{Radicular (Periapical) Cyst}

The radicular cyst is the most common acquired odontogenic cyst that arises from epithelial cell rests of periodontal ligament. This cyst is usually associated with tooth infection and nonvital tooth. ${ }^{3}$ On CT, it appears as a unilocular radiolucent periapical lesion with well-defined, sclerotic borders in close vicinity of the apical portion of the root of a nonvital carious tooth, most commonly the upper lateral incisor. ${ }^{3,10}$ Residual cyst is the periapical cyst retained in the jaw after surgical removal of a nonvital tooth. ${ }^{3}$

\section{Dentigerous (Follicular) Cyst}

Dentigerous cysts are the most common developmental cysts of odontogenic origin, arising from the dental follicle of a developing or an unerupted tooth., ${ }^{2,3}$ They are typically diagnosed in the age group of 20 to 40 years. When large, these cysts show significant buccolingual expansion and thinning of cortices with predisposition to infection or pathological fracture. On CT, they appear as a well-circumscribed unilocular pericoronal area of radiolucency with sclerotic borders around the crown of an unerupted tooth, typically the lower third molar fol-

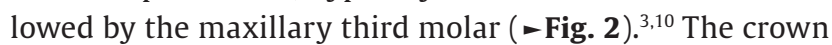
generally protrudes into the cyst, whereas the root of the affected tooth is outside the lesion, and the distance between the crown and the dental sac is usually greater than $3 \mathrm{~mm}^{3}{ }^{3}$ Convergence of cyst walls at the cementoenamel junction is an important diagnostic clue. ${ }^{7}$



STEP-VI: Other lesion specific imaging features like cortical integrity, periosteal reaction, perilesional halo, growth pattern, root displacement or resorption and associated soft tissue are also looked for

Fig. 1 Stepwise imaging approach to pediatric jaw lesions on OPG and MDCT (OPG, orthopantomograph; MDCT, multidetector computed tomography; OKC, odontogenic keratocyst; CEOT, calcified epithelial odontogenic tumor; SBC, simple bone cyst; LCH, Langerhans cell histiocytosis; CGCG, central giant cell granuloma; ABC, aneurysmal bone cyst; JOF, juvenile ossifying fibroma; FD, fibrous dysplasia). 


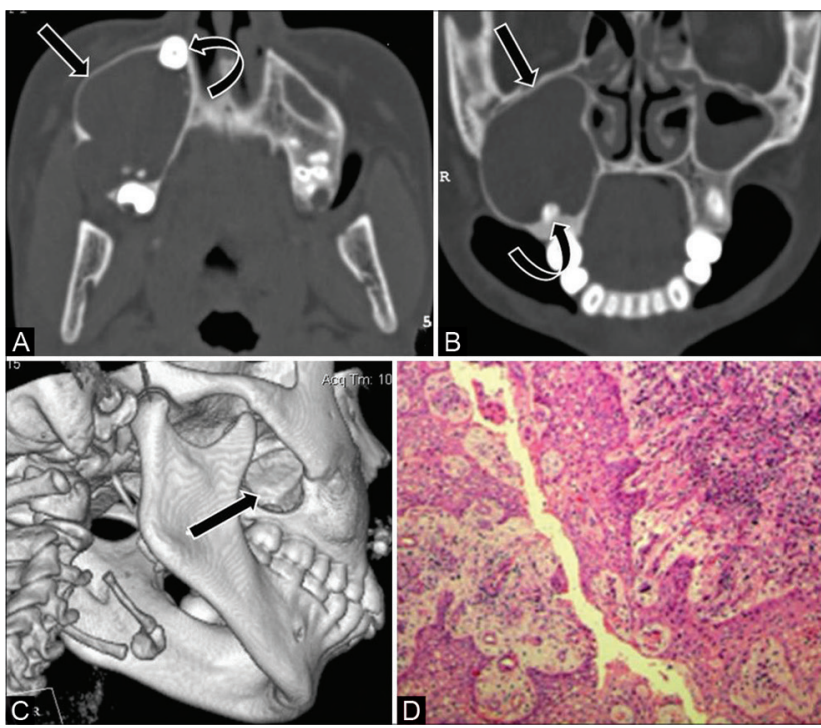

Fig. 2 Dentigerous cyst in a 14-year-old adolescent girl with swelling right upper jaw. (A) Axial computed tomography (CT) and (B) coronal multiplanar reconstruction (MPR) bone window reveal a unilocular, well-circumscribed radiolucent expansile lesion (arrow) surrounding the crown of an unerupted tooth (curved arrow) in the alveolar process of the right maxilla, encroaching upon the ipsilateral maxillary sinus. (C) 3D reformat demonstrates the margin of cyst (arrow). (D) Histopathological examination (HPE) reveals cyst lined by nonkeratinized stratified squamous epithelium confirming the diagnosis of dentigerous cyst.

\section{Odontogenic Keratocyst}

The odontogenic keratocyst (OKC) is now reclassified under the developmental odontogenic cyst. OKC was placed under the category of odontogenic tumors in the 2005 classification, due to its aggressive behavior.,11 It arises from the dental lamina and has a characteristic lining of uniform stratified squamous epithelium with palisading and overlying corrugated parakeratin layer. ${ }^{4} \mathrm{~A}$ high recurrence rate after surgery is seen because of aggressive and infiltrative behavior along with the presence of daughter cysts in the adjacent bone. ${ }^{3}$ OKC appears as unilocular or multilocular lytic lesion with well-corticated margins typically involving the posterior body or ascending ramus of the mandible. ${ }^{3,9,10}$ Important diagnostic clue is tendency of growth within the medullary space along the length of bone in an anteroposterior direction with minimal buccolingual expansion, which helps differentiation of a unilocular OKC with the crown of an unerupted tooth from the dentigerous cyst. ${ }^{7,10}$ Root resorption is uncommon, and weak enhancement of the uniformly thin and regular cyst walls with no obvious soft tissue helps differentiation from unilocular ameloblastoma. Multiple OKCs are seen in the Gorlin-Goltz syndrome ( - Fig. $\mathbf{3}$ ), Ehlers-Danlos syndrome, and Noonan's syndrome. In addition to multiple OKCs, the Gorlin-Golz syndrome shows basal cell nevi, skeletal abnormalities, mental retardation, and falx cerebri calcifications. ${ }^{3,10}$

\section{Ameloblastoma}

Ameloblastoma is a benign but locally invasive, slow-growing odontogenic tumor that arises from the enamel-forming

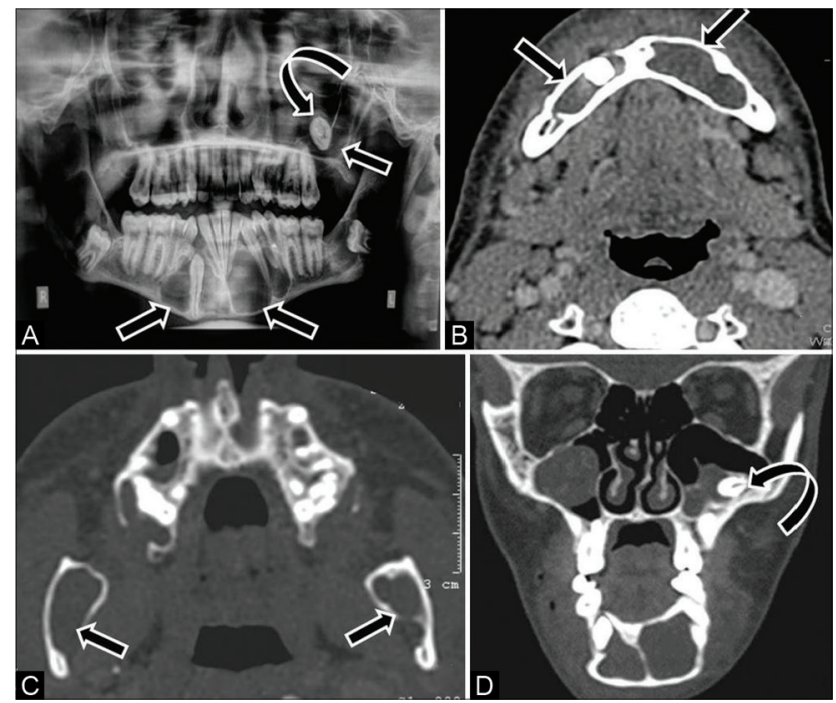

Fig. 3 Multiple odontogenic keratocysts in an 18-year-old male with Gorlin-Goltz syndrome. (A) Orthopantomograph (OPG) showing multiple radiolucent lesions (arrow) in mandible, displacement of teeth, and misplaced left third molar overlying the left maxillary sinus (curved arrow). (B,C) Contrast-enhanced computed tomography (CECT) scans of axial soft tissue and bone window show multiple unilocular cysts (arrow) with growth along the anteroposterior length of the jaw and no enhancing soft tissue. (D) Coronal multiplanar reconstruction (MPR) bone window shows cyst with crown of an unerupted tooth in the left maxilla (curved arrow). Noncontrast computed tomography (NCCT) of the brain showed falx cerebri calcification supporting the diagnosis of Gorlin-Goltz syndrome.

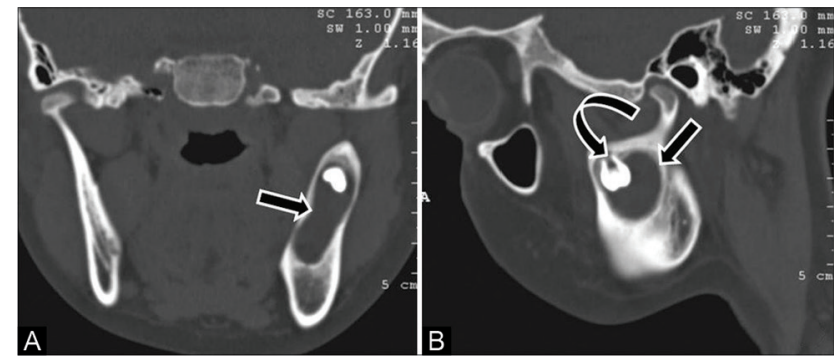

Fig. 4 A 17-year-old adolescent girl with painless swelling in the left lower jaw. (A) Cornonal computed tomography (CT) and (B) sagittal multiplanar reconstruction (MPR) bone window show unilocular expansile cystic lesion (arrow) with unerupted tooth (curved arrow) in the left ramus of mandible. Imaging diagnosis of dentigerous cyst was given. Histopathology confirmed dentigerous cyst with focal area of plexiform ameloblastoma.

cells of odontogenic epithelium. It can also arise from the pluripotential epithelial lining of an odontogenic cyst (5\%; - Fig. 4) ${ }^{1,10}$ Conventional ameloblastomas are typically seen in the third to fourth decade of life; however, unicystic forms can be seen in children and adolescent and show lower recurrence rate. The different histopathological subtypes of ameloblastoma are follicular (most common), plexiform, acanthomatous, granular cell, basaloid, and desmoplastic. ${ }^{4}$ Patients present with painless slow-growing mass most commonly in the posterior mandible (80\%), typically in the third molar region. On imaging, unicystic ameloblastoma appears as a unilocular well-corticated lucent lesion with significant buccolingual expansion and solid nodular soft-tissue 


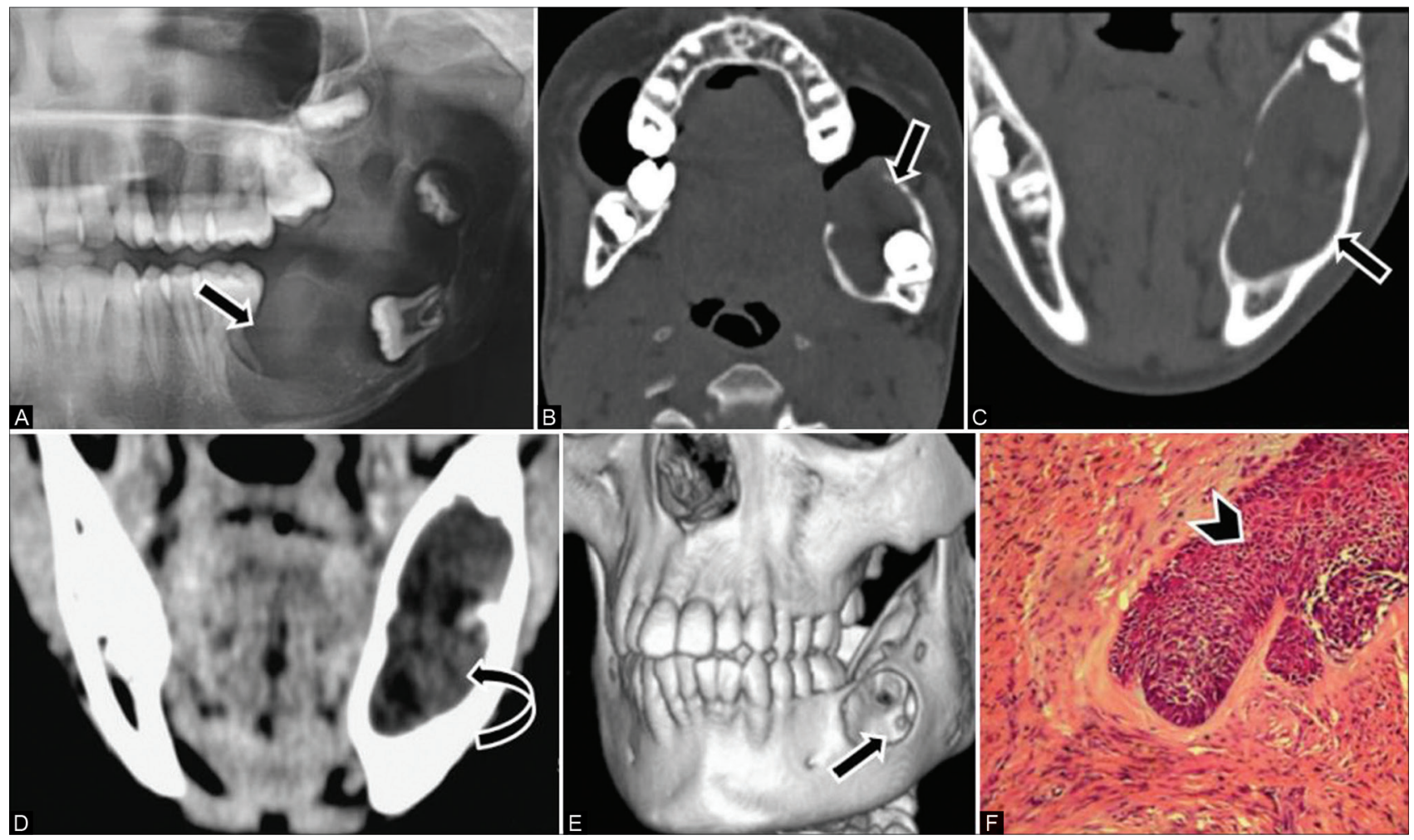

Fig. 5 Ameloblastoma in a 12-year-old boy with a swelling left lower jaw. (A) Orthopantomograph (OPG) showing a unilocular radiolucent lesion in left hemimandible (arrow). (A) Axial computed tomography (CT) and (C) coronal multiplanar reconstruction (MPR) bone window show unilocular expansile radiolucent lesion in the left posterior mandible in relation to the crown of an unerupted tooth (arrow) and thinning of the lingual cortex. (D) Contrast-enhanced CT (CECT) of coronal MPR soft-tissue window shows enhancing soft tissue (curved arrow). (E) 3D reformat shows the external extent of the lesion (arrow). (F) Histopathological examination reveals tumor cells arranged in follicles (arrowhead) separated by connective tissue stroma confirming the diagnosis.

component (-Fig. 5)-a feature that differentiates it from odontogenic keratocyst and dentigerous cysts., ${ }^{9,10,12}$ Larger lesions may cause tooth displacement or root resorption. Conventional ameloblastoma appears classically as multilocular expansile radiolucent lesions with a soap bubble or honeycomb appearance. .,10 $^{70}$

\section{Odontogenic Myxoma}

Odontogenic myxoma is a locally aggressive infiltrating benign true odontogenic tumor originating from the mesodermal portion of odontogenic apparatus.,10 It commonly occurs in 10- to 30-year-olds and presents as a slow-growing painless lesion with equal incidence in the maxilla and the mandible (ramus being most common mandibular site). ${ }^{10}$ Rapid growth of the tumor results in extensive osseous destruction and perforation of cortex with extension into surrounding soft tissues. ${ }^{1,10}$ On CT, it appears as a unilocular or multilocular lytic lesion separated by internal delicate bony trabeculae that form geographic compartments with a “tennis racket" appearance (-Fig. 6).,10,12 Root resorption and teeth displacement may occur.7,10 Appearance may resemble hemangioma, central giant cell granulomas (CGCGs), and ameloblastoma. However, MRI may help by showing T2 high signal intensities due to abundant myxoid stroma. On contrast administration, odontogenic myxoma shows a gradual enhancement pattern as against the avid enhancement in hemangioma. ${ }^{10,12}$ Histologically, they show hypocellular stellate cells in the myxoid background. ${ }^{4}$

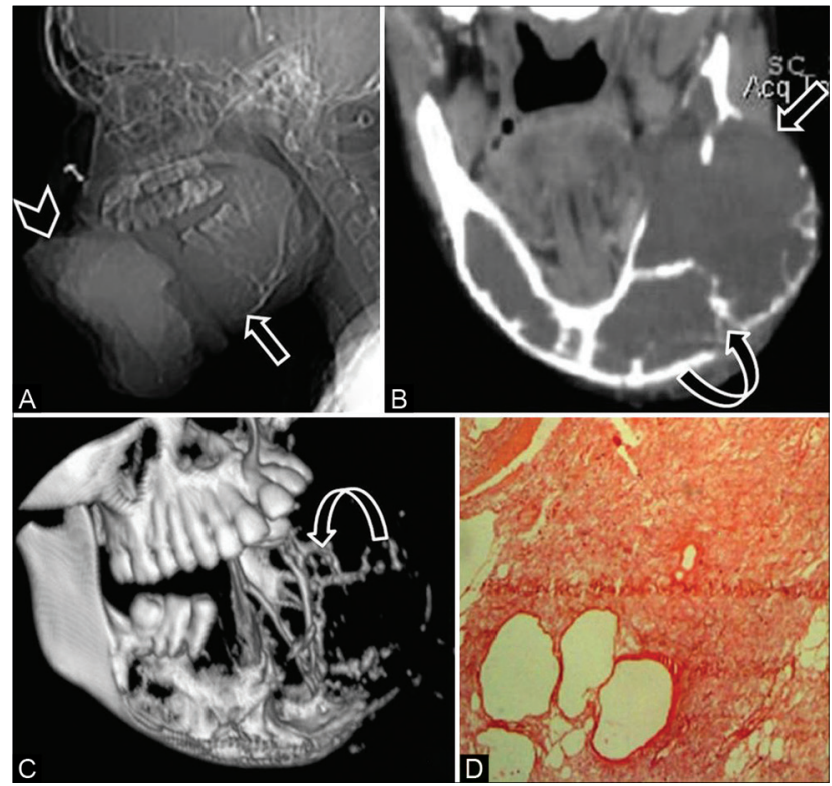

Fig. 6 Odontogenic myxoma in a 16-year-old adolescent girl with a large painful mass lower jaw. (A) Computed tomography (CT) scanogram shows a large soft-tissue density mass (arrowhead) and expansile lytic lesion in the mandible (arrow). (B) CT coronal multiplanar reconstruction (MPR) soft-tissue window shows a large lytic expansile lesion (arrow) with peripheral curved bony trabeculae-"tennis racket" appearance (curved arrow). (C) 3D reformat depicts the entire tumor extent and internal bony trabeculae (curved arrow). (D) Histopathological examination reveals stellate cells in myxoid background with few thin-walled vascular spaces confirming the diagnosis. 


\section{Radiopaque Lesions}

\section{Cementoblastoma}

Cementoblastomas are benign odontogenic periapical neoplasms that arise from the cementum in children and young adults. Fifty percent of patients with cementoblastoma present before the age of 20 years and $75 \%$ present before 30 years. $^{6}$ They commonly involve the mandible and are seen in association with the apex of the premolar or molars. Cementoblastomas are commonly associated with an erupted permanent tooth; however, they can be seen with an unerupted or impacted tooth. Imaging shows a round, periapical, sclerotic, sharply marginated sunburst mass attached to the root of the tooth with a low-attenuation thin radiolucent halo. ${ }^{6,9}$ It resembles osteoblastoma histologically; however, fusion of the tumor with the root of the tooth along with perpendicular orientation of the peripheral trabeculae to the capsule are characteristic features of cementoblastomas. ${ }^{4}$

\section{Odontomas}

Odontomas are the most common odontogenic tumors seen in children, and represent a developmental aberration (hamartoma). ${ }^{69}$ Approximately 50\% are associated with an impacted tooth, although they may develop after tooth eruption. ${ }^{9}$ Odontomas develop between the roots of the teeth and are classified as compound and complex types. Compound odontoma commonly occurs in the anterior maxilla and comprises multiple small radiographically identifiable tooth components (abortive teeth or denticles). Complex odontomas are seen as well-demarcated amorphous hyperattenuating conglomerate mass of enamel and dentin surrounding the crown of the tooth. ${ }^{2,4,6}$ Complex odontomas are commonly located in the molar regions of the jaws and are surrounded by a low-attenuation halo, a feature that allows differentiation from osteoma (-Fig. 7). ${ }^{6}$ Histologically, odontomas contain a mixture of acellular dentin containing tubules and enamel matrix. ${ }^{4}$

\section{Mixed-Density Lesions}

\section{Calcifying Epithelial Odontogenic Tumor (Pindborg's Tumor)}

Calcifying epithelial odontogenic tumor is a benign but locally aggressive tumor, uncommon in children. Typical location is in the premolar or the molar region of the mandible and most tumors are associated with the crown of an impacted tooth. CT shows radiolucent lesion with scattered calcified components and may show the impacted tooth within. ${ }^{1,9}$ Histologically, it is an epithelial tumor with fibrous stroma and extracellular amyloid protein having a tendency to calcify. ${ }^{4,9}$

\section{Cemento-Ossifying Fibroma}

Cemento-ossifying fibromas have been classified under odontogenic tumors in the latest 2017 WHO classification of odontogenic cysts and tumors, separate from juvenile ossifying fibromas (JOFs). These are benign mesenchymal
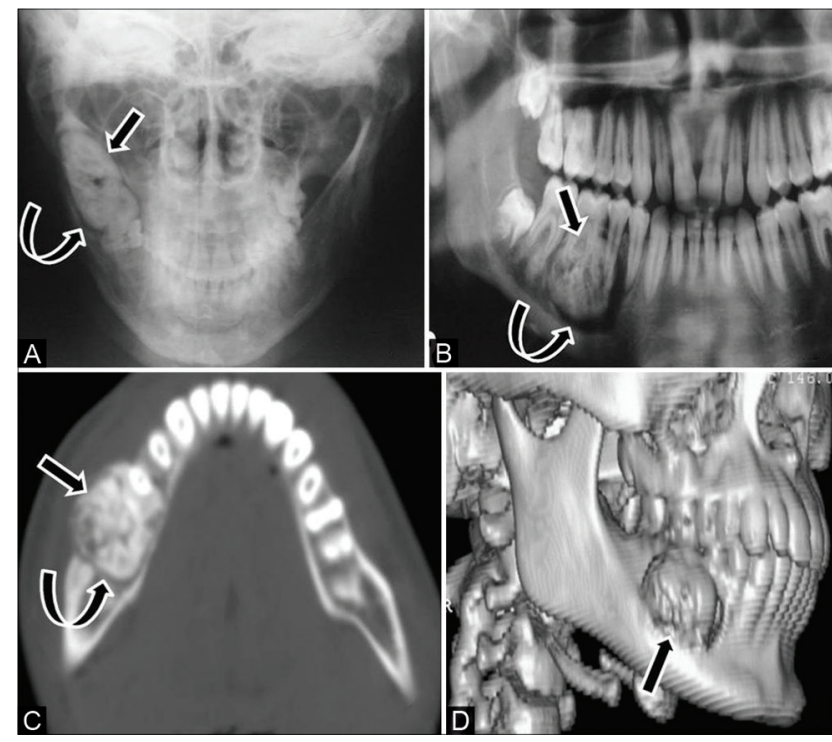

Fig. 7 Odontoma in a 16-year-old adolescent boy with mild swelling along the right lower jaw. (A) Plain radiograph and (B) OPG reveal a mildly expansile radiodense lesion (arrow) with a lucent rim (curved arrow) in the right mandibular ramus. (C) Computed tomography (CT) scans of axial bone window and (D) 3D reformat show mildly expansile conglomerated hyperattenuating amorphous mass (arrow), in relation to roots of premolar and first molar tooth with a characteristic low-attenuation halo (curved arrow) around it with no differentiation into individual tooth suggestive of complex odontoma.

odontogenic tumors arising within the periodontal ligament. The tumor comprises cellular fibroblastic stroma with varying amounts of bony trabeculae and cementumlike spherules. ${ }^{4,11}$ Cemento-ossifying fibromas become more radiopaque as they mature and appearance may resemble fibrous dysplasia. However, as against fibrous dysplasia, these lesions are well demarcated, and often encapsulated. ${ }^{13}$

\section{Nonodontogenic Lesions}

Nonodontogenic lesions usually have no specific relationship to dentition, can involve the bone around two or more teeth, and are seen below the inferior alveolar canal. - Table 2 shows the lists of nonodontogenic cysts and tumors of the jaw in children.

\section{Radiolucent Lesions}

\section{Simple (Solitary/Unicameral) Bone Cyst}

Simple bone cyst is usually an incidentally detected pseudocyst that arises due to posttraumatic intramedullary hemorrhage. ${ }^{3,10}$ Common age of presentation is 10 to 20 years with typical location in the marrow of the posterior mandible. ${ }^{3}$ On OPG, it appears as a unilocular low-density lesion with well-defined borders causing minimal bone expansion and a scalloped appearance of the superior margin of cyst between the tooth roots. ${ }^{2,3,10}$ No associated root resorption or tooth displacement is seen as this pseudocyst insinuates between teeth. CT and MRI provide information on the hemorrhagic content in the lesion and no postcontrast enhancement is seen. ${ }^{3}$ 
Table 2 Nonodontogenic cysts and tumors of jaw in children

\begin{tabular}{|l|}
\hline Nonodontogenic cysts \\
\hline Solitary bone cyst \\
\hline Aneurysmal bone cyst \\
\hline Stafne's bone cyst \\
\hline Nonodontogenic tumors \\
\hline Benign mesenchymal tumors \\
\hline $\begin{array}{l}\text { Giant cell lesions: giant cell tumor, central giant cell gran- } \\
\text { ulomas, brown tumor of hyperparathyroidism, cherubism }\end{array}$ \\
\hline $\begin{array}{l}\text { Fibro-osseous lesions: juvenile ossifying fibroma, fibrous } \\
\text { dysplasia, cemento-osseous dysplasia }\end{array}$ \\
\hline $\begin{array}{l}\text { Bone and cartilage tumors: osteoma, osteochondroma, } \\
\text { desmoplastic fibroma }\end{array}$ \\
\hline $\begin{array}{l}\text { Malignant mesenchymal tumors: Ewing's sarcoma, } \\
\text { osteosarcoma }\end{array}$ \\
\hline $\begin{array}{l}\text { Vascular tumors: intraosseous hemangioma, vascular } \\
\text { malformations }\end{array}$ \\
\hline $\begin{array}{l}\text { Hematopoietic and reticuloendothelial tumors: Burkitt's } \\
\text { lymphoma, non-Hodgkin's lymphoma, Langerhans cell } \\
\text { histiocytosis }\end{array}$ \\
\hline Neurogenic tumors: neuroblastoma \\
\hline
\end{tabular}


Fig. 8 Aneurysmal bone cyst in an 8-year-old girl with slow-growing painful left cheek mass. (A) Plain radiograph shows increased density over the left maxilla (arrow). (A) Axial computed tomography (CT) and (C) coronal multiplanar reconstruction (MPR) bone window show multilocular expansile lytic lesion in the left maxilla, with thin internal septa, ballooning of the cortex (curved arrow) extension into the alveolar margin, displaced teeth (arrowhead) and obliteration of maxillary sinus cavity (asterisk). (D) contrast-enhanced CT (CECT) coronal MPR soft-tissue window shows the entire extent of the lesion (curved arrow) with no associated soft tissue. Diagnosis of aneurysmal bone cyst $(A B C)$ was confirmed on histopathologic examination.

\section{Aneurysmal Bone Cyst}

$\mathrm{ABC}$ is an uncommon benign cystic jaw lesion with a spongelike appearance due to multiple blood-filled cavities. It can be locally aggressive due to its rapid growth and osteolytic tendency. ${ }^{14} \mathrm{ABC}$ is usually seen in patients younger than 20 years and more common in female, with the posterior mandible as the most common location. ${ }^{2,14}$ Common presentation is a painless (or dull pain) rapidly growing swelling, which can be disfiguring. Imaging shows a unilocular or multilocular expansile lytic lesion with a honeycomb or soap bubble appearance, causing expansion, ballooning, and destruction of the cortex and tooth displacement or root resorption. On $\mathrm{CT}, \mathrm{ABC}$ shows a typical meshwork appearance that reflects partially cystic, blood-filled meshwork divided by the coarse septa ${ }^{10,14,15}$ ( - Fig. 8). Contrast-enhanced images show enhancement of cyst wall and septa. Fluid-fluid levels (due to hemorrhage) on CT and MRI provide diagnostic confirmation. MRI helps differentiate $A B C$ from odontogenic myxoma, CGCGs, OKC, and hemangioma. ${ }^{10}$

\section{Central Giant Cell Granuloma}

CGCG is a benign non-neoplastic, occasionally aggressive intraosseous proliferative lesion predominantly affecting females in second and third decades of life.,16 Typical locations are the anterior maxilla and the mandible. On CT, it appears as a multilocular (less often unilocular) well-defined radiolucent lesion with thin internal septations (honeycomb appearance), usually in the anterior location and crosses the


adjacent teeth is an important diagnostic clue. ${ }^{2}$ Histologically, it shows presence of fibrous tissue, hemorrhage, and characteristic osteoclastlike giant cells. ${ }^{16}$ CGCG closely resembles brown tumors of hyperparathyroidism on imaging as well as
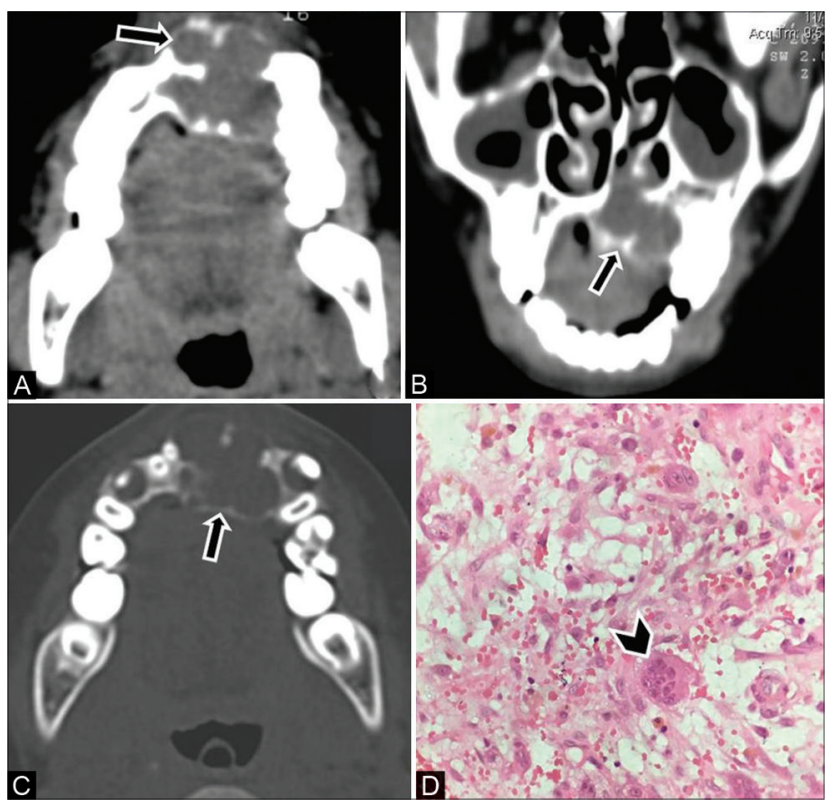

Fig. 9 Central giant cell granuloma of the maxilla in a 12-year-old boy. (A) Axial computed tomography (CT) and (B) coronal multiplanar reconstruction (MPR) soft-tissue window show a multilocular expansile lucent lesion (arrow) with scalloped border predominantly in the left anterior maxilla, crossing the midline. Thin internal septations are seen with a honeycomb appearance. (C) CT of the axial bone window shows similar multilocular appearance (arrow). (D) Histopathological examination shows multinucleated giant cells (arrowhead) in fibrovascular stroma, confirming the diagnosis. 
pathology; however, multifocal involvement and biochemical profile in hyperparathyroidism help in differentiation. ${ }^{7,13,16}$

\section{Langerhans Cell Histiocytosis}

Langerhans cell histiocytosis ( $\mathrm{LCH}$ ) is a rare benign disorder of reticuloendothelial system with pathologic proliferation of mononuclear histiocytic cells, the Langerhans cells resulting in local tissue infiltration and destruction. Eosinophilic granuloma is the most benign and localized form of LCH, generally seen in children younger than 15 years (males $>$ females). ${ }^{3}$ Bones commonly involved are skull, flat bones, spine, and long bones. Oral manifestations like inflammatory gingival hypertrophy or tooth loosening may be the first signs of $\mathrm{LCH}$, and involvement of the jaw is seen in $7.9 \%$ with typical location in the body and angle of the mandible. ${ }^{17}$ Imaging shows a well-marginated, localized, punched-out or scooped-out lytic lesion with or without reactive sclerosis ( - Fig. 10). ${ }^{17-19}$ Destruction of the alveolar bone and loss of lamina dura result in a characteristic "floating tooth" appearance. ${ }^{3,17}$

\section{Ewing's Sarcoma}

Ewing's sarcoma is the most aggressive primary malignant bone tumor in children that arises from undifferentiated osseous mesenchymal cells. Out of 4\% Ewing's sarcoma in the head and neck, $1 \%$ occur in the jaw with a predilection for the ramus of mandible (90\% primary lesion). ${ }^{20,21}$ Ewing's sarcoma is commonly seen in first two decades of life with male preponderance. ${ }^{21}$ Usual presentation is painful rapidly progressing swelling, loose teeth and nonspecific systemic

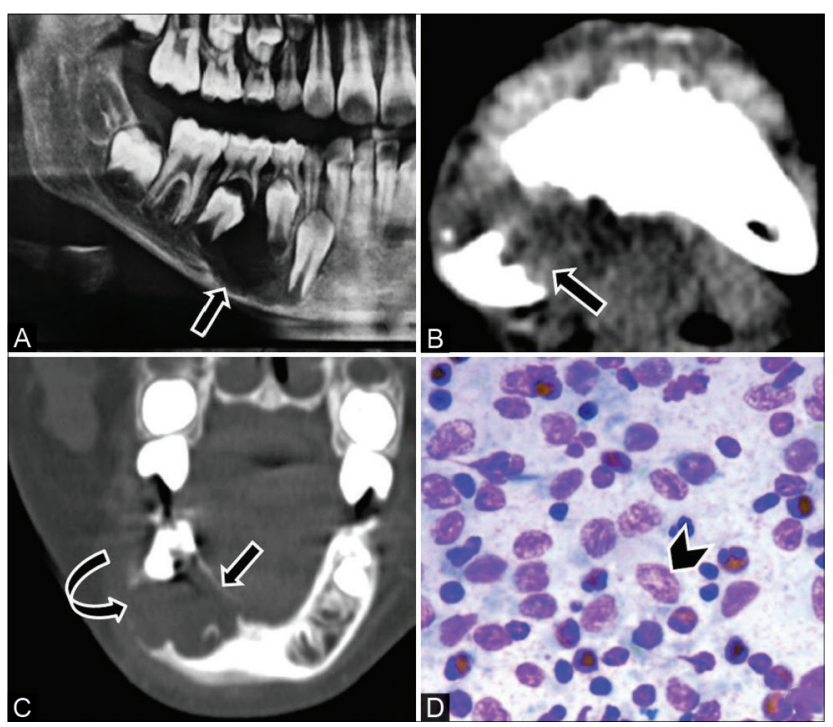

Fig. 10 Langerhans cell histiocytosis in a 9-year-old boy with a painful swelling right lower jaw, and clinical diagnosis of dentoalveolar abscess. (A) Orthopantomograph (OPG) shows a well-defined radiolucent lesion (arrow) in the right hemimandible adjacent to unerupted premolars. (B) Computed tomography (CT) of the axial soft-tissue window and (C) coronal multiplanar reconstruction (MPR) bone window show a single well-marginated scooped-out lytic lesion (arrow) in the right posterior mandible with areas of cortical thinning (curved arrow), with no periosteal reaction or associated soft tissue. (D) Cytopathology reveals Langerhans cells (arrowhead) in the background of the eosinophils and lymphocytes. symptoms. Histologically, it is a small round cell tumor with CD99 and MIC2 positive. ${ }^{20}$ OPG shows an ill-defined permeative osteolytic lesion; it may also show cortical destruction, root resorption, and displacement of teeth. CT additionally shows the extent and pattern of bone destruction, floating teeth appearance, associated enhancing soft-tissue mass, and typical sunray-type periosteal reaction ( - Fig. 11) ${ }^{18,21-23}$ Characteristic onion peel-type periosteal reaction of Ewing's sarcoma as seen elsewhere in the body is not seen because of typical flat bone with less mass of the mandible. ${ }^{22,23}$ Imaging differentials may include osteomyelitis and neuroblastoma metastases. ${ }^{20,21}$

\section{Burkitt's Lymphoma}

An aggressive form of non-Hodgkin's lymphoma, Burkitt's lymphoma is the least common type of lymphoma in all age groups. It is one of the fastest growing human malignancies with a doubling time of 24 to 48 hours; however, it is completely curable and highly sensitive to chemotherapy. ${ }^{24}$ Three clinical subtypes are seen: the endemic variant, sporadic variant, and immunodeficiency-associated Burkitt's lymphoma. Jaw and other facial bone involvement is more common in the endemic variant (associated with EpsteinBarr virus [EBV] infection), seen in Africa in malaria-endemic areas. The sporadic variant is associated with EBV infection in $30 \%$ of cases. It usually involves Waldeyer's ring, lymph nodes, and the abdomen; however, it can rarely involve the jaw bones..$^{24,25}$ On CT, Burkitt's lymphoma is seen as a

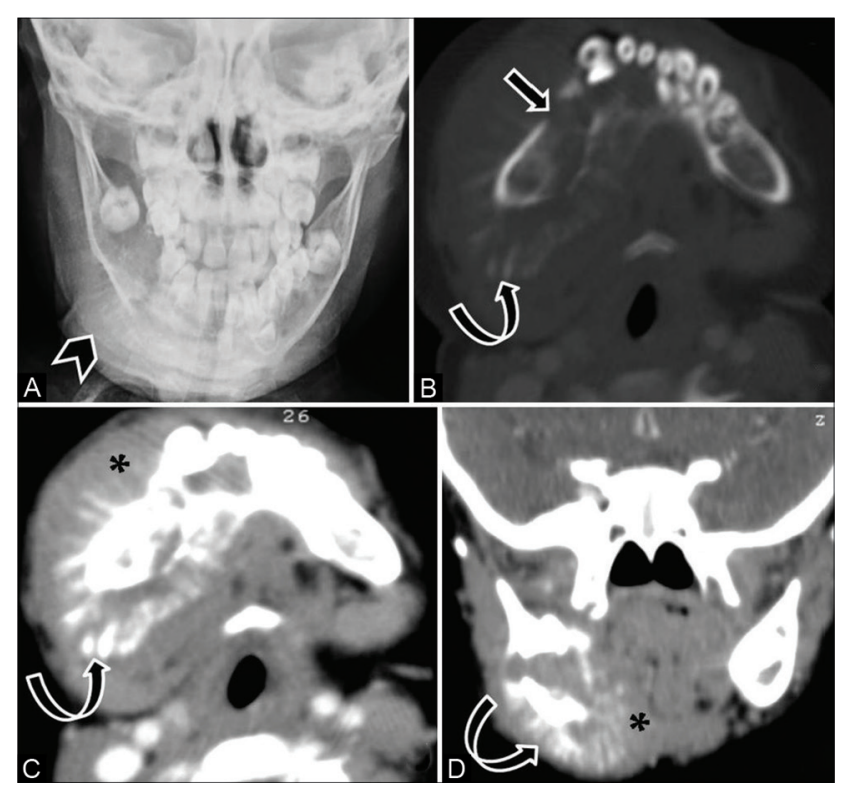

Fig. 11 Ewing's sarcoma of the right lower jaw in a 2-year-old girl. (A) Plain radiograph shows a lytic lesion in the right hemimandible, sunray periosteal reaction and adjoining soft tissue (arrowhead). (B) Computed tomography (CT) of the axial bone window shows ill-defined lytic expansile lesion (arrow), wide transition zone, destruction of body and ramus of right hemimandible with characteristic sunray periosteal reaction (curved arrow). (C) Axial contrast-enhanced CT (CECT) and (D) coronal multiplanar reconstruction (MPR) of soft-tissue window show significant enhancing soft-tissue (asterisk). CT of the chest showed a small metastatic nodule in the right middle lobe. Ewing's sarcoma was confirmed on histopathological examination. 
soft-tissue mass with massive bone destruction around the jaw causing loosening or displacement of teeth due to loss of lamina dura and cortical disruption ( - Fig. 12). MRI helps in assessment of marrow infiltration and submucosal extension of tumor. ${ }^{18,24,25}$ Differential diagnosis may include metastasis neuroblastoma and leukemia. Biopsy is mandatory for correct diagnosis before starting treatment. ${ }^{3}$

\section{Radiopaque Lesions}

\section{Exostoses}

Exostoses or tori are generally asymptomatic slow-growing benign localized bony outgrowths on the surface of the mandible or the maxilla. These are covered by a thin layer of poorly vascularized mucosa and show spontaneous arrest of growth. Exostoses are rarely seen in children and show four subtypes depending upon the site of origin. Torus mandibularis arises above the mylohyoid line, along the lingual surface of the mandible; buccal exostosis arises from the buccal cortex of the maxilla; torus palatinus arises midline on the hard palate; and torus maxillaris arises from the lingual surface of the posterior maxilla., 6,13

\section{Osteoma}

Osteoma is a benign bone tumor composed of mature compact or cancellous bone, commonly arising in craniofacial bones. It is uncommon in children, and the posterior mandibular body or condyle is the usual location. ${ }^{6}$ On imaging,

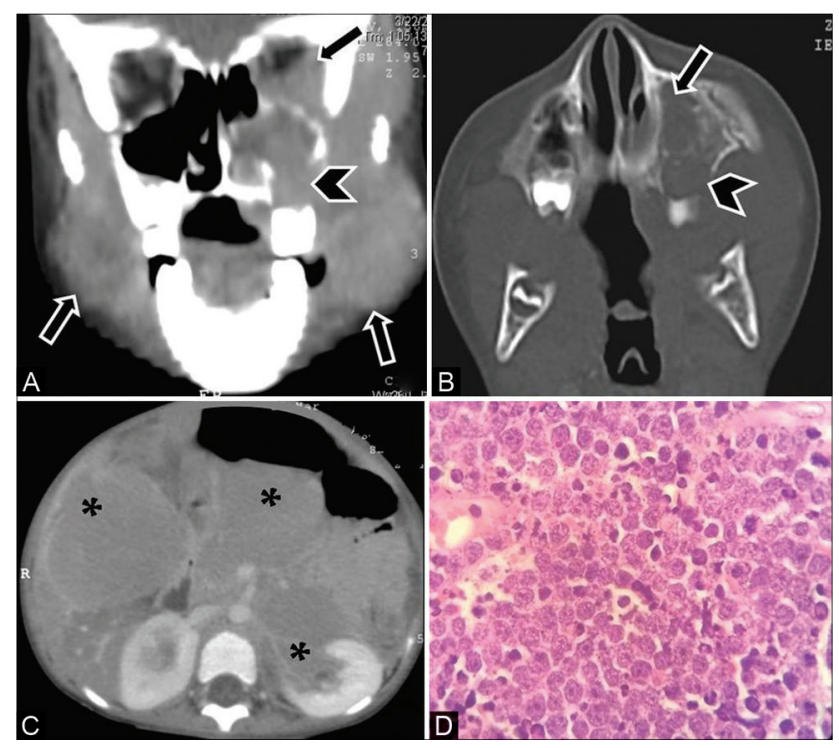

Fig. 12 Burkitt's lymphoma in a 4-year-old child with abdominal lump and facial swelling. Contrast-enhanced computed tomography (CECT) of the face (A) coronal multiplanar reconstruction (MPR) soft-tissue window (B) axial bone window show enhancing soft-tissue masses (arrow) in the masticator spaces, left maxillary sinus, nasal cavity, and orbit with ill-defined lytic lesions in the left maxilla (arrowhead). (C) CECT of the abdomen shows multiple intraperitoneal and retroperitoneal masses with vascular encasement, involvement of liver, pancreas, duodenum, and kidneys (asterisk). (D) histopathological examination reveals lymphocytes with scanty cytoplasm, "starry sky" appearance, and tangible-body macrophages consistent with diagnosis.
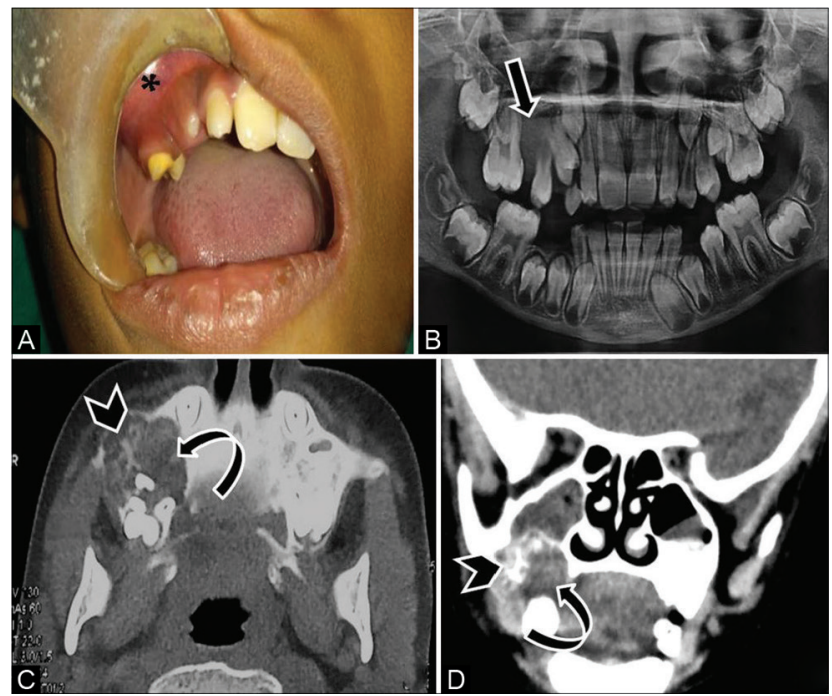

Fig. 13 Juvenile ossifying fibroma in an 11-year-old boy with pain and swelling right mid-face. (A) Clinical picture shows gum swelling in the right upper jaw (asterisk). (B) Orthopantomograph (OPG) shows a well-bordered radiodense expansile lesion in the right maxilla (arrow). Computed tomography (CT) of the (C) axial bone window and (D) coronal multiplanar reconstruction (MPR) soft-tissue window show an expansile mixed-density lesion with narrow zone of transition, ground-glass appearance (curved arrow) and few areas of calcification (arrowhead). Juvenile ossifying fibroma was confirmed on histopathological examination.

it appears as a well-circumscribed sclerotic mass with no perilesional halo and causes mild bone expansion. Multiple osteomas may be associated with Gardner's syndrome.,

\section{Mixed-Density Lesions}

\section{Juvenile Ossifying Fibroma}

JOF is an uncommon, fibro-osseous lesion with aggressive behavior and tendency to recur. Histologically, two variants are seen, the psammomatoid and trabecular JOFs. Psammomatoid subtypes are more common in adults with the orbit and the paranasal sinuses being the predominant locations. The trabecular subtype more commonly occurs in the jaw, and shows a high tendency to recur. ${ }^{11,18,26}$ The trabecular JOF is typically seen in boys younger than 15 years. CT shows a solitary expansile well-defined lesion with ground-glass attenuation, varying degree of calcification, thin sclerotic border, and no low-attenuation halo (-Fig. 13). Developing tooth can be displaced, missing, or remain unerupted. ${ }^{1,6,18}$ Histologically, it is similar to cement-ossifying fibroma comprising of cellular fibrous stroma, bony strands, and cement particles $(4,26)$.

\section{Fibrous Dysplasia}

Fibrous dysplasia is an idiopathic developmental dysplastic disorder, where medullary bone is replaced by fibro-osseous tissue. It usually presents in childhood or in the early adolescence period with predominant involvement of the maxilla.,27 It can be monostotic (70\%) or polyostotic (30\%) and shows a heterogeneous ground-glass attenuation (homogenous in more mature forms). The characteristic features are bony 


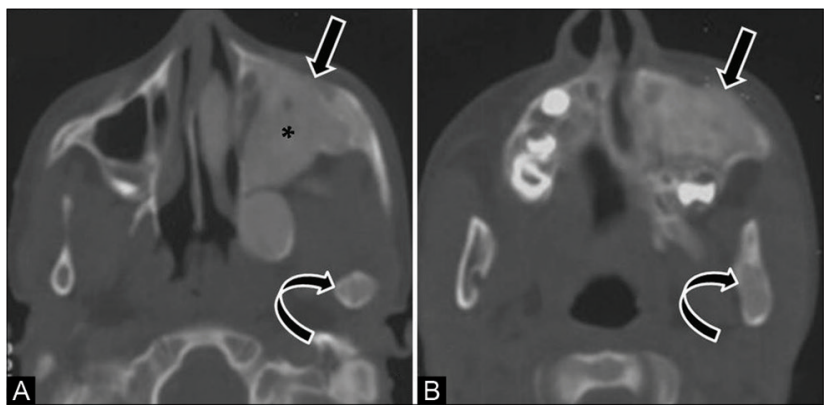

Fig. 14 Fibrous dysplasia with jaw involvement in a 9-year boy with a swelling left-side face. (A,B) Computed tomography (CT) scans of the axial bone window show an ill-defined expansile lesion in the left maxilla (arrow) and ramus of mandible (curved arrow) with heterogeneous ground-glass attenuation, wide transition zone, and intact cortex. Involvement of the left maxillary alveolar process with complete obliteration of the left maxillary antrum (asterisk) is seen. Expansion and involvement of other facial and cranial bones in the left side with ground-glass attenuation was also seen, suggestive of polyostotic craniofacial fibrous dysplasia.
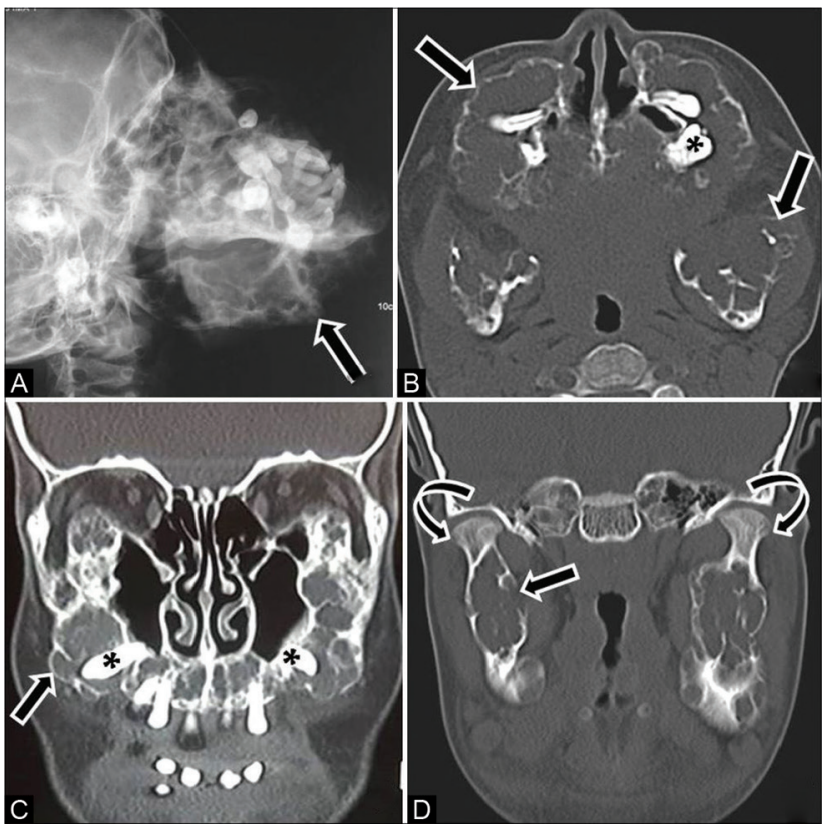

Fig. 15 Cherubism in an 11-year-old girl with painless progressive swelling of the jaw since 2 years of age. (A) Skull lateral radiograph shows gross expansile bone remodeling with multiple radiolucent areas in bilateral maxilla and mandible (arrow), causing displacement of teeth. Computed tomography (CT) of the (B) axial and (C,D) coronal multiplanar reconstruction (MPR) soft tissue and bone window show multilocular expansile lytic lesions with coarse trabecular bony pattern involving the entire maxilla, body, and ramus of mandible (arrow) along with displacement of teeth (asterisk), but preservation of the mandibular condyles (curved arrow).

expansion along the longitudinal axis of the affected bone, cortical thickening or thinning with intact cortex, and osseous deformity with no periosteal reaction ( - Fig. 14) $)^{2,6}$ Important diagnostic clues that help in differentiation of fibrous dysplasia from ossifying fibroma are ill-defined lesions, wide transition zone, longitudinal growth pattern, nondisplacement of the teeth, and crossing of sutures. In addition, fibrous dysplasia does not show osteoblastic rimming seen in ossifying fibroma on pathology. ${ }^{6}$

\section{Cherubism}

Cherubism is a rare, inherited developmental disorder with clinical and radiographic similarity to craniofacial fibrous dysplasia. However, it shows a tendency toward spontaneous remission and is a separate entity on histopathology and genetic analysis. Typical presentation is a bilateral relatively symmetrical painless expansion of the jaw with a predilection for ramus of the mandible or tuberosity of maxilla, and pathognomonic sparing of the mandibular condyle. ${ }^{2}$ Bone expansion starts in early childhood and grows progressively until puberty and shows gradual resolution thereafter. On imaging, variable-sized multilocular lucent areas with expansile bone remodeling, thinning of cortices, internal bony trabeculations, and dental derangement are seen. These lesions do not show periosteal reaction or associ-


imaging appearance rarely needs biopsy for diagnosis; however, pathology shows multinucleated giant cells in fibrous connective tissue stroma. ${ }^{2,7,28}$

\section{Intraosseous/Central Hemangioma}

Central hemangioma is a benign vascular tumor composed of disorganized masses of endothelial-lined spaces filled with blood. Common locations are in the vertebrae and skull with rare involvement of the jaw, usually involving the molar region of the mandible. ${ }^{23}$ It is usually seen in the second decade of life and has a nonspecific imaging appearance. OPG may show a lesion with ground-glass attenuation. CT shows a well-defined expansile, multilocular lesion with

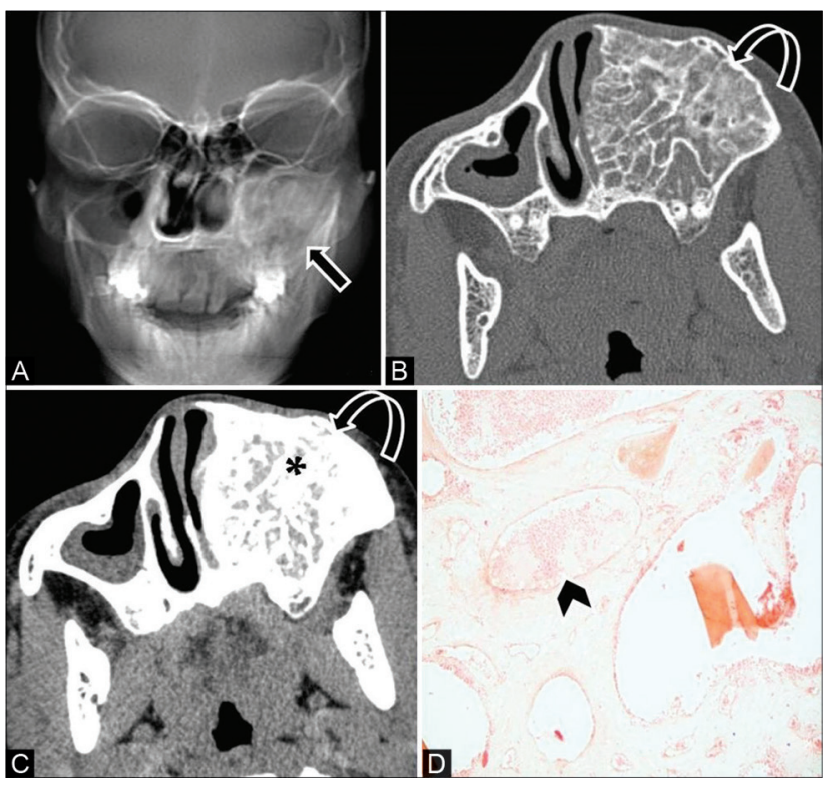

Fig. 16 Intraosseous hemangioma in a 14-year-old adolescent girl with a painless swelling on the left side of the face. (A) Reconstructed skull radiograph shows ground-glass opacity (arrow) overlying the left maxillary sinus and upper jaw. (B) Noncontrast computed tomography (NCCT) axial bone window shows multiseptated expansile honeycomb lesion with coarse trabeculae (curved arrow), intervening ground-glass density involving the alveolar ridge of the left maxilla. (C) Contrast-enhanced CT (CECT) of the axial soft-tissue window shows intense enhancement of lesion (asterisk). (D) histopathological examination reveals variable size vascular spaces, ectatic vessels, areas of hemorrhage (arrowhead), separated by bony trabeculae. 
small radiolucent locules separated by coarse bony trabeculae giving a honeycomb and soap bubble appearance (-Fig. 16).2,23,29 Displacement of adjacent teeth and resorption of tooth roots are often seen. ${ }^{29}$ Contrast-enhanced CT shows marked enhancement of the lesion that helps in differentiation from other multilocular radiolucent lesions like $A B C$, central giant cell reparative granuloma, and odontogenic myxoma..$^{23,29}$

\section{Osteomyelitis of Jaw}

Rare involvement of the jaw (1-6\%) by osteomyelitis is attributed to paucity of cancellous bone; however, the mandible (alveolar and angle region) is involved more than the maxilla because of its rich blood supply. ${ }^{6,30}$ It is usually seen in children younger than 16 years (60\% cases). ${ }^{31}$ Modes of infection may be (1) direct extension from pulpal infection in carious tooth, an extraction wound, or perforation of an erupting tooth; (2) regional extension of soft-tissue lesion to involve the underlying bone; and (3) hematogenous route. ${ }^{31}$ Acute forms (usually bacterial origin) are diagnosed clinically, shows response to antibiotic treatment, and can result in chronic osteomyelitis if inadequately treated. However, chronic forms of osteomyelitis can also occur due to long-term low-grade infection like tuberculosis, which may remain clinically silent., ${ }^{2,3}$ Cross-sectional imaging is needed to define extent and severity of lesion and to identify soft-tissue extension or associated abscesses. On imaging, acute osteomyelitis shows an ill-defined area of osteolysis
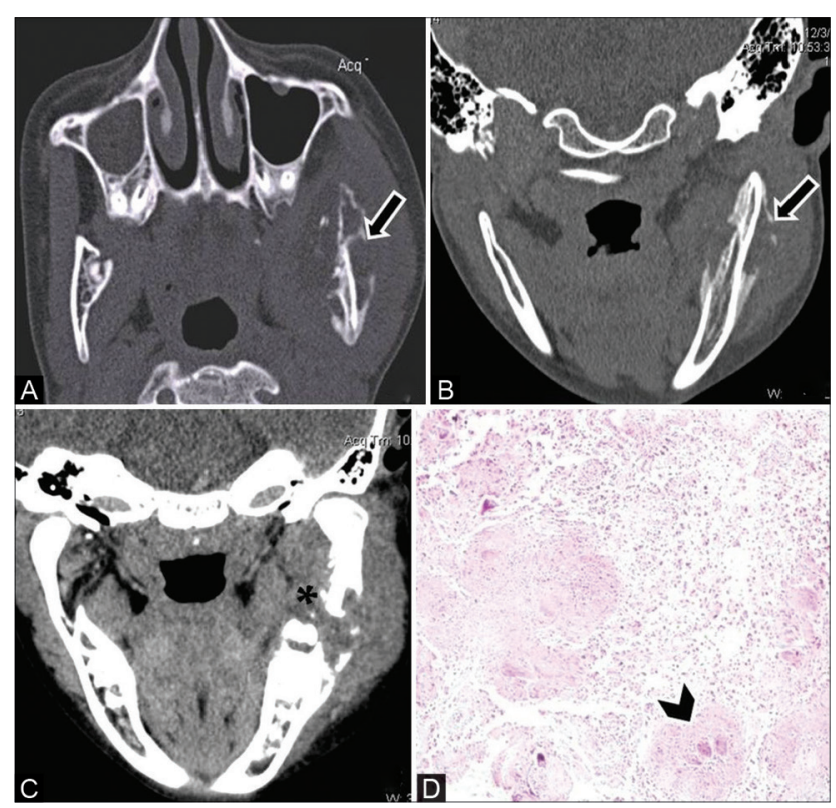

Fig. 17 Tubercular osteomyelitis in a 12-year-old boy with diffuse hard swelling left face and positive contact history of tuberculosis (TB). (A) Axial computed tomography (CT) and (B) coronal multiplanar reconstruction (MPR) bone window show osteolytic lesions (arrow) causing destruction of both inner and outer cortices in the body and ramus of the left mandible with periosteal reaction. (C) Contrast-enhanced CT (CECT) coronal reformatted soft-tissue window reveals adjacent muscle and soft-tissue involvement with heterogeneous enhancement (asterisk). (D) Histopathological examination shows epithelioid cell granuloma with Langerhans giant cells (arrowhead) confirming the diagnosis of tubercular osteomyelitis. with periosteal reaction and associated soft-tissue changes like cellulitis, myositis, and abscess. ${ }^{2}$ Chronic osteomyelitis appears as mixed lytic-sclerotic or lytic destructive lesion having ill-defined borders with blurring of trabecular details. Periosteal new bone formation, sequestrum, associated enhancing soft tissue, and fistula with discharging sinus may be seen (- Fig. 17) ${ }^{2,30,31}$ MRI is more sensitive in detecting bone marrow and soft-tissue involvement. ${ }^{3}$

\section{Osteosarcoma}

Osteosarcoma, a highly malignant bone tumor, is rare in jaw comprising less than $10 \%$ of all osteosarcomas. ${ }^{22}$ Although osteosarcoma commonly affects the long bones in the second decade of life, occurrence in jaw bones is usually seen in 30- to 40-year patients. ${ }^{22,32}$ The posterior mandible is the most common site of involvement, and symmetrical widening of the periodontal membrane may be the earliest radiographic finding of osteosarcoma. Low-grade osteosarcoma shows lytic destruction with ill-defined margins, whereas in the osteoblastic form (common in the jaw), a sclerotic bone lesion with radiating mineralized tumor spicules, sunburst appearance, and small amount of associated soft tissue may be seen. ${ }^{7,22}$

\section{Secondary Involvement of the Jaw}

\section{Metastasis to the Jaw}

Involvement of the mandible is more common than involvement of the maxilla in metastatic diseases with the propensity for angle and posterior body due to increased marrow space and vascularity. ${ }^{9}$ Common malignancies that metastasize to the jaw in children are neuroblastoma, leukemia, lymphoma, and Ewing's sarcoma. ${ }^{18}$ Involvement of leukemia may present with loosening of teeth due to loss of lamina dura, and lytic bone destruction may be seen later. ${ }^{13}$ On CT, neuroblastoma metastases are seen as ill-defined lytic lesions with a moth-eaten appearance (-Fig. 18) and sun ray-type periosteal reaction. ${ }^{18,33}$

\section{Direct Neoplastic Extension}

Locally aggressive soft-tissue tumors in the vicinity of the jaw may involve the maxilla and the mandible and osseous destruction. This is seen as an ill-defined geographic,



Fig. 18 Metastatic neuroblastoma to the jaw in a 6-month-old infant with abdominal distension and jaw swelling. Contrast-enhanced computed tomography (CECT) scans of the $(\mathbf{A})$ axial jaw bone window and (B) soft-tissue window show multiple ill-defined lytic lesions with a moth-eaten appearance (arrow) involving the left maxilla and mandible with associated enhancing soft tissue (arrowhead). CECT of the abdomen $(\mathbf{C})$ axial section reveals a large ill-defined heterogeneously enhancing left suprarenal mass (curved arrow) suggestive of neuroblastoma with lymph node and liver metastasis (asterisk). 

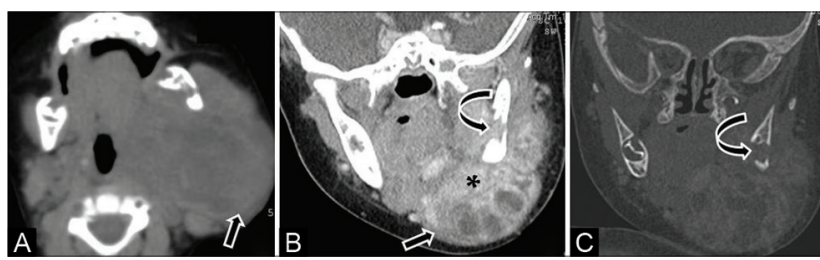

Fig. 19 Involvement of the mandible in soft-tissue hemangiopericytoma in a 5-month-old infant with rapidly progressing swelling left cheek and upper neck soon after birth, with no history of birth trauma. (A) Noncontrast computed tomography (NCCT) of the axial, (B) contrast-enhanced CT (CECT) coronal multiplanar reconstruction (MPR) soft-tissue window, and (C) CT coronal MPR bone window show a large heterogeneous infiltrative multispatial mass (arrow) predominantly in the left submandibular region abutting the left hemimandible. Mass shows intense contrast enhancement (asterisk) and a lytic destructive lesion in the body of the left hemimandible (curved arrow) is seen.
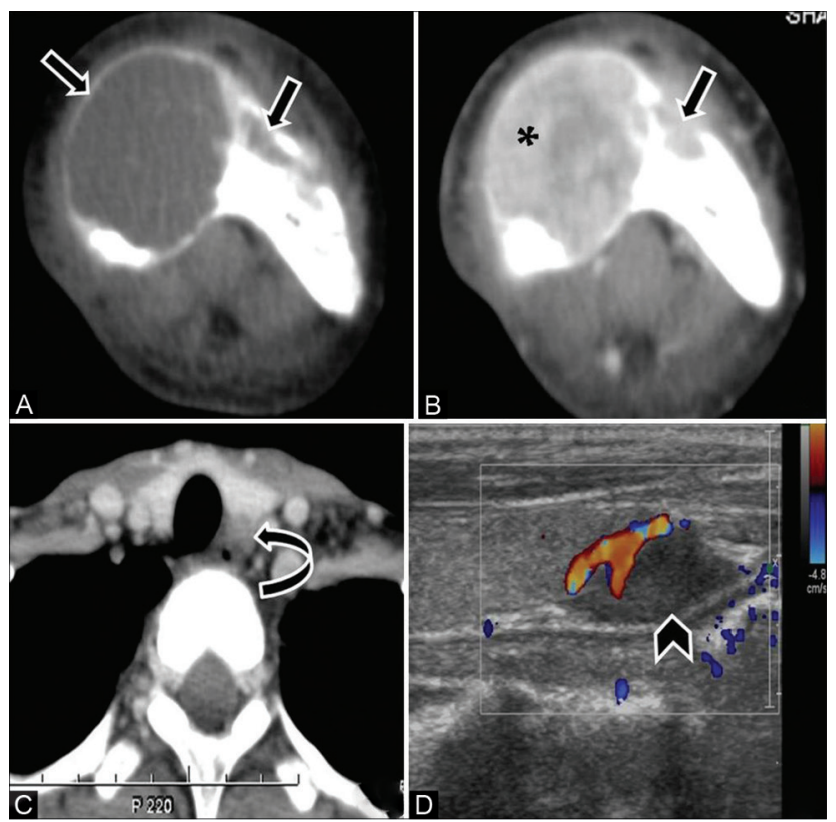

Fig. 20 Brown tumors in an 18-year-old female with a painful jaw swelling. (A) Noncontrast computed tomography (NCCT) and (B) contrast-enhanced CT (CECT) axial soft-tissue window show expansile variable-sized lytic lesions (arrow) in the mandible with granular texture (asterisk). (C) CECT neck shows a small hypodense mass in the left tracheoesophageal groove posteromedial to the left lobe of thyroid (curved arrow). (D) On ultrasonography (USG), this mass is seen in relation to the inferior pole of the left thyroid (arrowhead) and shows internal vascularity suggestive of parathyroid adenoma. Biochemical analysis revealed elevated serum calcium and parathyroid hormone (PTH) level, confirming diagnosis of hyperparathyroidism.

moth-eaten, or permeative bone destruction adjacent to soft-tissue tumor. ${ }^{12}$ These tumors may include rhabdomyosarcoma, salivary gland tumors, and hemangiopericytoma in the pediatric population ( - Fig. 19).

\section{Association of Jaw Lesions with Other Diseases}

\section{Brown tumors of Hyperparathyroidism}

Brown tumors are non-neoplastic, reactive focal bony lesions due to bone remodeling caused by long-standing hyperparathyroidism. They are more common in females and usually involve the ribs, clavicle, and pelvic girdle; they are rarely seen in the maxillofacial region (involvement of mandible commoner than maxilla). ${ }^{34}$ They appear as focal lesions with both osteoclastic and osteoblastic activities causing bone resorption with fibrous replacement of the marrow and thinning of the cortex. CT shows multifocal variable-sized well-defined lytic lesions that may produce cortical expansion ( - Fig. 20). In addition, bone changes due to hyperparathyroidism, for instance, generalized demineralization of jaw bones and loss of lamina dura, may also be seen. Biochemical parameters along with ultrasonography (USG) of the parathyroid gland help in achieving the correct diagnosis. ${ }^{34-36}$

\section{Jaw involvement in Tuberous Sclerosis}

The jaw lesions associated with tuberous sclerosis may include gingival fibroma, angiofibroma, odontogenic myxoma, desmoplastic fibroma, calcified odontogenic tumor, and intraosseous hemangioma. ${ }^{37}$ Desmoplastic fibroma is a rare, locally aggressive bone tumor of myofibroblastic origin that represents the intraosseous counterpart of the soft-tissue desmoids tumor or fibromatosis. ${ }^{23,37}$ It usually involves the mandible, tibia, scapula, and femur, and is commonly seen in childhood and adolescence with female preponderance. ${ }^{37,38}$ On CT, it appears as an expansile unilocular or multilocular lytic lesion with narrow transition zone, no matrix mineralization, marked cortical thinning, and often delayed enhancing soft tissue representing the fibrous nature of the lesion. Tumor extension into the surrounding soft tissues indicates a locally aggressive behavior. Desmoplastic fibroma has a high recurrence rate even after local resection and needs differentiation from other lytic lesions..$^{23,37-39}$

\section{Myxofibrosarcoma}

Myxofibrosarcoma is a myxoid variant of malignant fibrous histiocytoma having greater than $50 \%$ myxoid component (as per WHO 2013 classification) with a diffusely infiltrative pattern, and a high chance of local recurrence. It is rarely seen in children with uncommon involvement of the head and neck regions (3\%). ${ }^{40}$ Clinical presentation is a painless enlarging

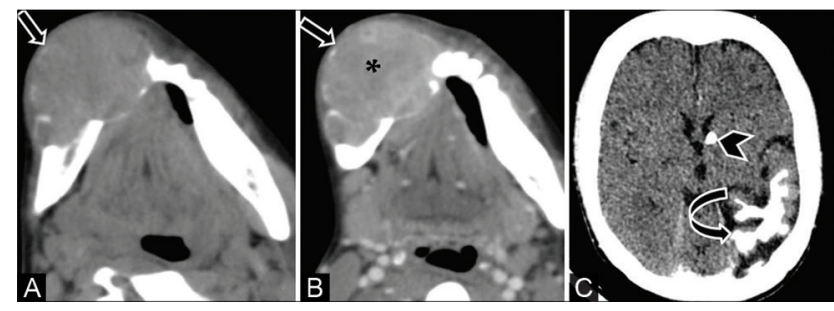

Fig. 21 A 15-year-old adolescent girl with tuberous sclerosis complex (TS) and mandibular swelling. (A) Noncontrast CT (NCCT) and (B) contrast-enhanced CT (CECT) axial soft-tissue window show a large expansile lytic lesion in the body of the right hemimandible with cortical thinning (arrow). Lesion shows narrow transition zone, few bony septa, no matrix mineralization, loss of ipsilateral canine tooth, and enhancing soft tissue within (asterisk). (C) NCCT of the brain shows focal cerebral atrophy, gyral calcifications of the left temporo-parieto-occipital region (curved arrow) and calcified subependymal nodule (arrowhead). In view of TS, imaging diagnosis of desmoplastic fibroma was given. 




Fig. 22 The same patient as in Fig. 21 presented after 18 months with increaseinjawswelling for which magneticresonanceimaging(MRI) was done. MRI mandible (A) axial T1-weighted image shows intermediate signal intensity lesion with few hypointense areas within (white star). (B) Axial and (C) sagittal T2-weighted images show a predominantly hypointense lesion (white star) with areas of hyperintensity within (white thin arrow) and perilesional T2-weighted hyperintensities in adjacent tissues likely due to edema. On histopathological examination, the lesion was confirmed as myxofibrosarcoma.

soft-tissue mass in the jaw. CT shows a hypoattenuating lesion and MRI is superior to CT for evaluation of tumor composition (-Figs. 21 and 22). Tumor shows low to intermediate signal intensity on T1-weighted images and mixed intensity on T2 weighted images (hypointense fibrous tissue with hyperintense myxoid component) with the enhancement of the solid soft-tissue component. ${ }^{40}$ Imaging features in favor of sarcomatous changes are size greater than $5 \mathrm{~cm}$, heterogeneous $\mathrm{T} 1$ and $\mathrm{T} 2$ signal intensity, and high $\mathrm{T} 2$ signal intensity of edematous surrounding tissues. ${ }^{41}$

\section{Conclusion}

Many jaw lesions, whether in a child or adult, have similar imaging appearance, and achieving a definitive diagnosis on imaging is a big challenge. A systematic approach to the evaluation of a child is required taking into consideration the age, gender, and clinical presentation along with the imaging features. While interpreting the imaging characteristics, attention must be given to the anatomical location and the relation of the lesion with the tooth and the inferior alveolar canal. It may not always be possible to reach a specific diagnosis; however, with a systematic evaluation, the list of differential diagnosis becomes narrow and more relevant.

\section{Financial Support and Sponsorship}

Nil.

\section{Conflict of Interest}

There are no conflicts of interest.

\section{Acknowledgment}

The authors would like to thank Professor (Dr.) Sapna Singh, MD Radiodiagnosis, for her contribution to images of $\boldsymbol{\imath}$ Figs. 14 and 15.

\section{References}

1 Akay MC, Zeytinoğlu M, Simşek B, Aras I, Multidisciplinary management of benign jaw tumors in children. In: Motamedi $\mathrm{MH}$, ed. A Textbook of Advanced Oral and Maxillofacial Surgery. Vol. 2. London: IntechOpen; 2015 273-304
2 Gadodia A, Seith A, Sharma R, Choudhury AR, Bhutia O, Gupta A. Multidetector computed tomography of jaw lesions in children and adolescents. J Med Imaging Radiat Oncol 2010;54(2):111-119

3 Avril L, Lombardi T, Ailianou A, et al. Radiolucent lesions of the mandible: a pattern-based approach to diagnosis. Insights Imaging 2014;5(1):85-101

4 Soluk-Tekkeşin M, Wright JM. The World health organization classification of odontogenic lesions: a summary of the changes of the 2017 (4th) edition. Turk Patoloji Derg 2018;34

5 El-Naggar AK, Chan JKC, Grandis JR, Takata T, Slootweg P, Odontogenic and maxilofacial bone tumours. In: El-Naggar AK, ed. WHO Classification of Head and Neck Tumours. 4th ed. Lyon: IARC; 2017 205-260

6 Curé JK, Vattoth S, Shah R. Radiopaque jaw lesions: an approach to the differential diagnosis. Radiographics 2012;32(7):1909-1925

7 Neyaz Z, Gadodia A, Gamanagatti S, Mukhopadhyay S. Radiographical approach to jaw lesions. Singapore Med J 2008;49(2):165-176, quiz 177

8 Kalkur C, Halim N, Sattur A, Burde K, Naikmasur V. Radiographical approach to multilocular radiolucent lesions of the jaws: a review. EC Dent Sci 2019;18:410-420

9 Dunfee BL, Sakai O, Pistey R, Gohel A. Radiologic and pathologic characteristics of benign and malignant lesions of the mandible. Radiographics 2006;26(6):1751-1768

10 Devenney-Cakir B, Subramaniam RM, Reddy SM, Imsande H, Gohel A, Sakai O. Cystic and cystic-appearing lesions of the mandible: review. AJR Am J Roentgenol 2011;196(6, Suppl):WS66-WS77

11 Speight PM, Takata T. New tumour entities in the 4th edition of the World Health Organization classification of head and neck tumours: odontogenic and maxillofacial bone tumours. Virchows Arch 2018;472:331-339

12 Bernaerts A, Vanhoenacker FM, Hintjens J, et al. Tumors and tumor-like lesions of the jaw: radiolucent lesions. JBR-BTR 2006;89(2):81-90

13 Harmon M, Arrigan M, Toner M, O'Keeffe SA. A radiological approach to benign and malignant lesions of the mandible. Clin Radiol 2015;70(4):335-350

14 López-Arcas Calleja JM, Cebrián Carretero JL, González Martín J, Burgueño M. Aneurysmal bone cyst of the mandible: case presentation and review of the literature. Med Oral Patol Oral Cir Bucal 2007;12(5):E401-E403

15 Shete A, Chavan M, Diwan N, Shete M. Aneurysmal bone cyst: rarity in mandible and its ambiguity with central giant cell granuloma. J Orofac Sci 2012;4:51-55

16 Nackos JS, Wiggins RH III, Harnsberger HR. CT and MR imaging of giant cell granuloma of the craniofacial bones. AJNR Am J Neuroradiol 2006;27(8):1651-1653

17 Can IH, Kurt A, Ozer E, Sarı N, Samim E. Mandibular manifestation of Langerhans cell histiocytosis in children. Oral Oncol Extra 2005;41:174-177

18 Becker M, Stefanelli S, Rougemont AL, Poletti PA, Merlini L. Non-odontogenic tumors of the facial bones in children and adolescents: role of multiparametric imaging. Neuroradiology 2017;59(4):327-342

19 Golai S, Nimbeni B, Patil SD, Kakanur M, Paul S. Langerhans histiocytosis in a child - diagnosed by oral manifestations. J Clin Diagn Res 2015;9(4):ZD09-ZD11

20 Krishna KB, Thomas V, Kattoor J, Kusumakumari P. A radiological review of Ewing's sarcoma of mandible: a case report with one year follow-up. Int J Clin Pediatr Dent 2013;6(2):109-114

21 Lopes SL, Almeida SM, Costa AL, Zanardi VA, Cendes F. Imaging findings of Ewing's sarcoma in the mandible. J Oral Sci 2007;49(2):167-171 
22 Weber AL, Bui C, Kaneda T. Malignant tumors of the mandible and maxilla. Neuroimaging Clin N Am 2003;13(3):509-524

23 Theodorou DJ, Theodorou SJ, Sartoris DJ. Primary non-odontogenic tumors of the jawbones: an overview of essential radiographic findings. Clin Imaging 2003;27(1):59-70

24 Derinkuyu BE, Boyunağa Ö, Öztunalı Ç, et al. Imaging features of Burkitt lymphoma in pediatric patients. Diagn Interv Radiol 2016;22(1):95-100

25 Kalisz K, Alessandrino F, Beck R, et al. An update on Burkitt lymphoma: a review of pathogenesis and multimodality imaging assessment of disease presentation, treatment response, and recurrence. Insights Imaging 2019;10(1):56

26 Keles B, Duran M, Uyar Y, Azimov A, Demirkan A, Esen HH. Juvenile ossifying fibroma of the mandible: a case report. J Oral Maxillofac Res 2010;1(2):e5

27 MacDonald-Jankowski DS. Fibro-osseous lesions of the face and jaws. Clin Radiol 2004;59(1):11-25

28 Beaman FD, Bancroft LW, Peterson JJ, Kransdorf MJ, Murphey MD, Menke DM. Imaging characteristics of cherubism. AJR Am J Roentgenol 2004;182(4):1051-1054

29 Mittal A, Sheikh SA, Vinod VC, Garg P. Images: central hemangioma of the maxilla. Indian J Radiol Imaging 2007;17:163-165

30 Koul PA, Khan UH, Jan RA, Shah TH, Bagdadi F, Shah S. Tubercular osteomyelitis of the mandible in a young female. Int J Mycobacteriol 2014;3(2):155-157

31 Gupta KB, Manchanda M, Yadav SP, Mittal A. Tubercular osteomyelitis of mandible. Indian J Tuberc 2005;52:147-150
32 Wang S, Shi H, Yu Q. Osteosarcoma of the jaws: demographic and CT imaging features. Dentomaxillofac Radiol 2012;41(1):37-42

33 Wade G, Revels J, Hartman L, Brown W. Pediatric mandibular metastasis: a rare finding of neuroblastoma. Radiol Case Rep 2017;13(1):289-294

34 Scarano A, Sinjari B, Artese L, Fanali S, Carinci F. Mandible brown tumor caused by primary hyperparathyroidism. Eur J Inflamm 2011;9:101-104

35 Scholl RJ, Kellett HM, Neumann DP, Lurie AG. Cysts and cystic lesions of the mandible: clinical and radiologic-histopathologic review. Radiographics 1999;19(5):1107-1124

36 Xie C, Tsakok M, Taylor N, Partington K. Imaging of brown tumours: a pictorial review. Insights Imaging 2019;10(1):75

37 Tandon S, Garg RK. Intraoral desmoplastic fibroma: a manifestation of tuberous sclerosis. Fetal Pediatr Pathol 2012;31(4):195-201

38 Fahmy MD, Gupta A, Padilla RJ, Segura A, Brookes CD. Desmoplastic fibroma associated with tuberous sclerosis: case report and literature review. Oral Surg Oral Med Oral Pathol Oral Radiol 2019;128(2):e92-e99

39 Konikoth S, Bose T, Anupama IV, Yogidas R. Desmoplastic fibroma of the mandible: a rare benign tumor mimicking malignancy. J Indian Acad Oral Med Radiol 2019;31:74-78

40 Roland CL, Wang WL, Lazar AJ, Torres KE. Myxofibrosarcoma. Surg Oncol Clin N Am 2016;25(4):775-788

41 Chhabra A, Soldatos T. Soft-tissue lesions: when can we exclude sarcoma. ? AJR Am J Roentgenol 2012;199(6):1345-1357 